\title{
Determination of Factors and Quality Control of Car Painting Based on FMEA and SPC.V2
}

\author{
Masud Rana1, Xinmin Zhang1, Sayed Abdul Akher ${ }^{2}$ \\ ${ }^{1}$ Department of Mechanical Engineering, Shenyang University of Technology, Shenyang, China \\ ${ }^{2}$ Department of Plant Genome Engineering, University of Electronic Science and Technology of China, Chengdu, China \\ Email: ranamasud.su@yahoo.com
}

How to cite this paper: Rana, M., Zhang, X.M. and Akher, S.A. (2018) Determination of Factors and Quality Control of Car Painting Based on FMEA and SPC.V2. Modern Mechanical Engineering, 8, 158-177. https://doi.org/10.4236/mme.2018.82011

Received: April 16, 2018

Accepted: May 27, 2018

Published: May 30, 2018

Copyright $\odot 2018$ by authors and Scientific Research Publishing Inc. This work is licensed under the Creative Commons Attribution International License (CC BY 4.0).

http://creativecommons.org/licenses/by/4.0/

\begin{abstract}
The global automotive industry demands world-class levels of product quality, productivity, and competitiveness as well as continual improvement. To achieve this goal, many vehicle manufacturers companies use quality control tools to improve the quality of the product with zero defects and highly satisfied to the customer. Nowadays, there are a lot of quality tools applied to solve the problem quickly but it's still the fact to find out good and efficient solving way. The study emphasizes the identification of potential failure which failures may have encountered in the production process and it will lead to car scrap, rework and influence of the internal production and quality target. After the complete study of the manufacturing process and production data-failure causes, failure rate and data etc. FMEA discover the weak processes in the form of higher risk priority number in the manufacturing of the car painting process, which required reducing by identifying and implementing of the defects and this will improve the process quality of the painting surface of the car. To analysis, the improvement of the car surface defects SPC (Statistical Process Control) tools are more efficient where can easily visible the defects trends. SPC chart is chronological graphs of process data that are used in manufactures industries to help understand, control and improve the process and that although based on statistical theory area easy for practitioners to use and interpret. In order to orient goal of zero defects of the car, surface uses the PFMEA technique to prioritize the defects and statistically analyze the roots cause of the defect and control the defects through continues improvements process.
\end{abstract}

\section{Keywords}

Quality Management, FMEA, SPC, Control Technology, Quality Improvement, Paint 


\section{Introduction}

Quality control and management is a crucial matter in the car industry. Considering the incessantly increasing requirements for the quality of products and process, it is necessary to improve a quality-oriented management in all types of manufacturing company [1]. In addition to diverse technical requirements are also considering the requirements of the national, international and company-specific norms. The company must not only fulfill the requirements of the quality, but also the requirement of safety, environment, and economy [2]. As following, some aspects of the manufacturing quality management and their integration manufacturing process will be introduced. Usually, technological advance will lead to process improvement with time and could ultimately approach the states of the Zero-defect. In the tremendous, competition, survival of the fittest is the law of competition in the market. The manufacturers need to use what kind of products, entirely by the market to make a fair conclusion [3]. Automated products have high demand and market orientation due to its high quality and low price. In such climate conditions, automobile production quality, efficiency, resilience has become hard in pursuit of the goal of the manufacturers and the increasingly fierce market competition, forcing the manufacturers must continue to introduce new varieties while meeting the needs of users [4]. In order to quickly seize the market, car manufacturers must be more variety, fast-paced, high-quality mixed production, what products the market needs, we can as fast as the best products to the market. In an automotive company, Paint shop top coat inspection line is one of the important areas to produce the car smoothly without major defects formation. It's very common fact that, in the top coat inspection line can highly influence the production rate and efficiency if there is no control of the defects and solving of the root cause of the defects origination [5]. For rational and effective use of topcoat inspection line, we must solve the problem and must control the defects to get the high-quality results of the automotive cars.

In addition, there is a lot of research in the quality control field to improve the production rate, reduction of the cost and improve the efficiency. The research is not limited only the domestic also huge development in the overseas [6]. There is a lot of quality control methods has been studied during the several decades but in the automotive company the research application related to practices [7]. In order to get the better quality of the product, there is only continuous improvement process and reduce the repair rate in the automotive company [8]. For the automotive company, it's very important to find out the root cause of each defect and print out a long-term solution because the defects are very sensitive in the car surface. So, after researching the many research papers, here will introduce the new way to find out the defects solutions [9] [10]. For the defects analysis, here will use the FMEA methodology to find out the most priority of the defects and use the fishbone diagram to analyze the origin of the defects [11]. After figure out the main reason for the defects, there will use SPC tool identify 
the performance trends. This factor is combined to assign prioritization for SPC implementation. This exercise that should be monitored on a periodic basis is via an enhanced method.

\section{Theoretical Framework}

\subsection{Failure Mode and Effects Analysis (FMEA)}

Failure Mode and Effects Analysis (FMEA) has long been used as a planning tool during the development of processes, products, and services. In developing the FMEA, the team identifies failure modes and actions that can reduce or eliminate the potential failure from occurring [4]. Input is solicited from a broad group of experts across design, test, quality, product line, marketing, manufacturing, and the customer to ensure that potential failure modes are identified. The FMEA is then used during deployment of the product or service for troubleshooting and corrective action. The standard FMEA process evaluates failure modes for occurrence, severity, and detection. The multiplication of these values leads to what is known as the risk priority number (RPN).

$$
R P N=\text { Occurrence } * \text { Severity } * \text { Detection }
$$

FMEA is a reliability tool, which requires identifying failure modes of a specific product or system, their frequency, and potential causes. According to Arinez, Biller, Meerkov, \& Zhang [12], the life cycle of a product is analyzed by an inter-functional work team. Daimler Chrysler, Ford, and General Motors have jointly developed an international standard named SAE J1739-2006 documentation for FMEA. This document provides general guidance in the application of different types of FMEA. First, the potential failure modes and potential causes are identified along with its effects and then the current controls are determined [13]. FMEA method is used to calculate RPN for each failure mode and then proposed recommended actions to reduce the RPN. The basic steps are to identify the root causes and potential problems that could occur, and then derive RPN which can direct improvement effort to the areas of greatest concern. Actions are then undertaken to reduce the risk presented by the failure mode. FMEA was developed at Grumman Aircraft Corporation in the 1950 and 1960s and it was first applied to the naval aircraft flight control systems at Grumman. Since, then, it has been extensively used as a powerful technique for system safety and reliability analysis of products and processes in a wide range of industries. Xiuxu Zhao presented a new approach for enterprises which combined Statistical Process Control (SPC) with FMEA knowledge library.

FMEA Primarily quality planning tool. It is used to develop features and goals for product and process, in identifying critical of product/process factor, designing customaries the potential problems, establishing the control to prevent the errors and prioritizing the process submit to ensure reliability [14]. FMEA most commonly applied but not limited to design (DFMEA) and manufacturing process (PFMEA). Design failure mode and effect analysis (DFMEA) identify the potential failure of design before they occur. DFMEA then goes to establish a 
potential effect of the failures, there causes, how often and when they might occur and their potential seriousness. Process failure mode and effect analysis (PFMEA) is systemized group of activities intended to recognized and evaluated the potential failure of a product/process and its effect identify action which could eliminate or reduce the occurrence or improve the defect ability, document the process and track change to avoid the potential failure cause. FMEA is carried out by a cross-functional team of experts from various departments [15]. Normally, a team is formed at the planning stage of a new product based on a concurrent engineering approach. The team analyzes each component and subsystem of the product for the failure modes. Then, the potential causes and effects are determined. The risk of each failure is prioritized based on the risk priority number (RPN). RPN is a decision factor based on three ratings: Severity $(\mathrm{S})$, Occurrence $(\mathrm{O})$ and Detection $(\mathrm{D})$. These ratings are scaled with numbers between 1 and 10. The analysis starts from the basic structure of the system and particularly from those system elements for which accurate information about failure mode and its causes are available. By analyzing the functional relationships among these elements, it is possible to identify the possibility of propagation of each type of failure to predict its effects on the production performance of the entire system [16]. This is an inductive method to analyze failure modes using down-top methodology. The FMEA is a formalized but subjective analysis for the systematic identification of possible root causes and failure modes and the estimation of their relative risks. The main goal is to identify and then limit or avoid risk within a design. Hence, the FMEA drives towards higher reliability, higher quality and enhance safety. FMEA concentrates on identifying the severity and criticality of failures. FMEA is a fully bottom-up approach. Risk Priority Number, which is the product of the severity, occurrence, and detection ratings are calculated as $R P N=S \times O \times D$. The RPN must be calculated for each cause of failure. $R P N$ shows the relative likelihood of a failure mode, in that the higher number, the higher the failure mode [17]. From $R P N$, a critical summary can be drawn up to highlight the areas where the action is most needed. The RPN is re-calculated after the failure has been addressed. The revised $R P N$ confirms the effectiveness of the corrective actions undertaken.

\subsection{Implementation Methods for FMEA}

Implementation starts with the FMEA planning and crosses function team and creation for FMEA development and the evaluation of the results. After preparation of the team and planning next step is to delay the manufacturing process and identification of each step process and documentation in the FMEA sheet. Standard FMEA sheet is developed by the IATF (International Automotive Task Force) which is given below (Figure 1):

Usually, it breaks the scope into separate subsystems, items, parts, assemblies or process steps and identify the function of each. Process identification characteristics come from the process diagram. A product characteristic is a feature 


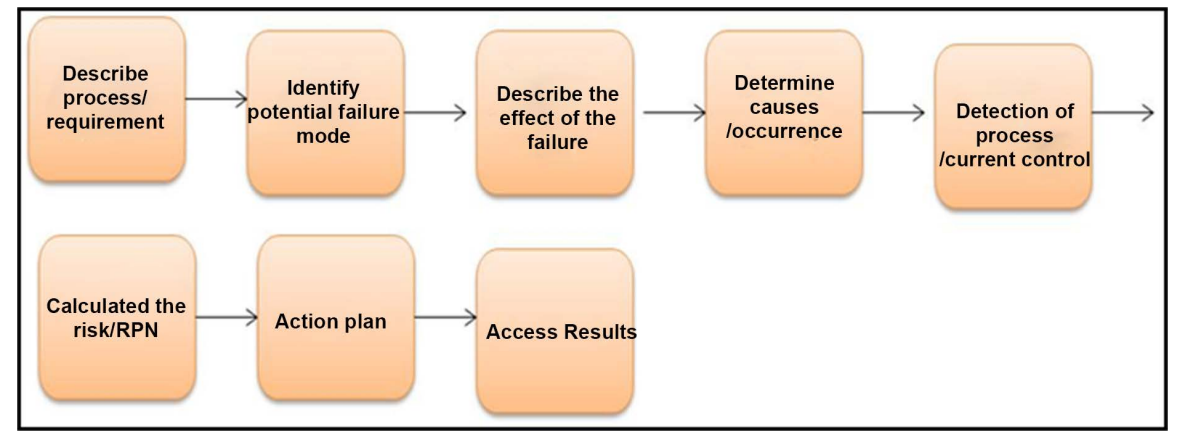

Figure 1. Basic process steps of the process FMEA.

such as dimension, size, form, location, orientation, location, texture, coating, hardness, strength, appearance, reflectivity.

\subsection{Potential Failure Mode}

For each function, identify all the ways failure could happen. These are potential failure modes. If necessary, go back and rewrite the function with more detail to be sure the failure modes show a loss of that function. Potential failure modes have defined the manner in which the process could potentially fail to meet the process requirement. It's a description of a non-conference at the specific operation [18]. It can be associated with a potential failure mode in the subsequent (downstream) operation or effect associated with a rotation failure in a process operation. However, preparation of FMEA, the assumption may be made that the incoming part/materials are correct.

\subsection{Potential Effect of Failure}

The potential effect of failure is defined as the effect of the failure mode on the customer. The customer in this content could be next operation, subsequent operation or location, the dealer, the vehicle owner. Each must be considered when assessing the potential effect of failure.

\subsection{Severity}

Severity is an assessment of the seriousness of the effect and refers directly to the potential failure mode being studied. The customer in process FMEA is both internal and where appropriate, the external customer. The severity ranking is also an estimate of how difficult it will be for the subsequent operation to be carried out to its specification its performance, cost and time [19]. The ranking and suggested criteria are based on IATF manual of FMEA version 3. A common industry standard scale uses 1 to represent no effect and 10 to indicate very much severe with failure affecting system operation and safety without warning.

\subsection{Cause of Failure Mode}

Identify the cause of each failure mode. A failure cause is defined as a design weakness that may result in a failure. The potential causes for each failure mode 
should be listed in technical terms and not in terms of symptoms. Examples of potential causes included improper torque applied, improper operating conditions, too much solvent, improper alignment, excessive voltage.

\subsection{Occurrence}

The occurrence is the assessment of the probability that the specific cause of the failure mode will occur. A numerical weight should be assigned to each because that indicates how likely that cause is probability of the occurrence. For that failure history is helpful increasing the truth of probability.

\subsection{Detection}

The detection steps distinguish between two steps of detection. On one hand to identify the current control process. The current control process is a mechanism that prevents the cause of the failure mode from occurring or which defect the failure before it reaches the customer. The engineer should now identify testing analysis, monitoring and other techniques that can or have been used on the same or similar products/process to detect the failure. The other things are to assess the probability that the proposed process controls will detect a potential cause of failure or a process weakness [7]. Assume the failure has occurred and then assess the ability of the control to prevent the shipment of the part with that defect, low occurrence does not mean low detection. The control should detect the low occurrence. The risk priority number is a mathematical product of the numerical severity, probability, and detection rating.

$$
R P N=(\text { severity } * \text { occurrence } * \text { detection })
$$

The $R P N$ is used to prioritize items that require addition quality planning activities. If the $R P N$ number high that means the occurrence of the failure is high.

\subsection{Actions}

Determine recommended action to address potential failures that have a high RPN. These actions could include specific of different components or materials, de-rating, limiting environmental stresses or operating range, redesign of the item to avoid the failure mode, monitoring mechanisms and inclusion of backup system (Figure 2).

At the input collection and analysis by the SPC trends of the defects frequency. After analyzing the defects priority use the FMEA tools to identify the root cause of the defects and get the FMEA repository [20]. Finally takes the process control of the defects. From the figure (Figure 3) we can understand the quality management module in the paint shop.

From Figure 4 shows the Quality gate in the different working station and offline checking of the care and section audit area in the paint shop. During the production, if there is any deviation of the process parameters or equipment instant can figure out the root cause from the quality gate and continue checking till is back to the normal batch production [13]. Each working station has the 
FMEA analysis knowledge management

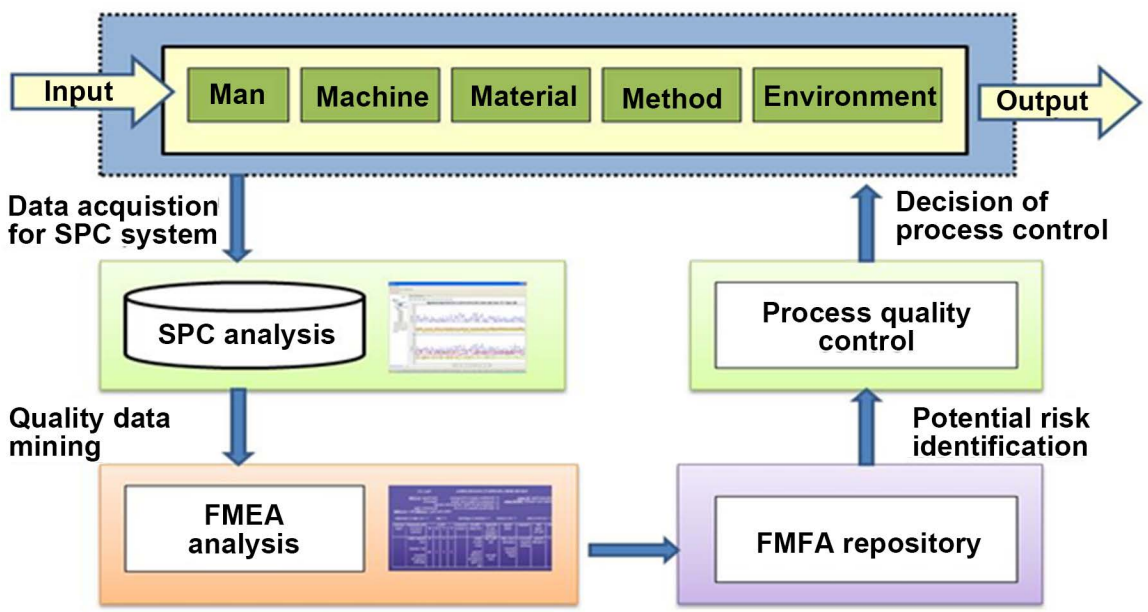

Figure 2. The principle of quality control based on FMEA and SPC.

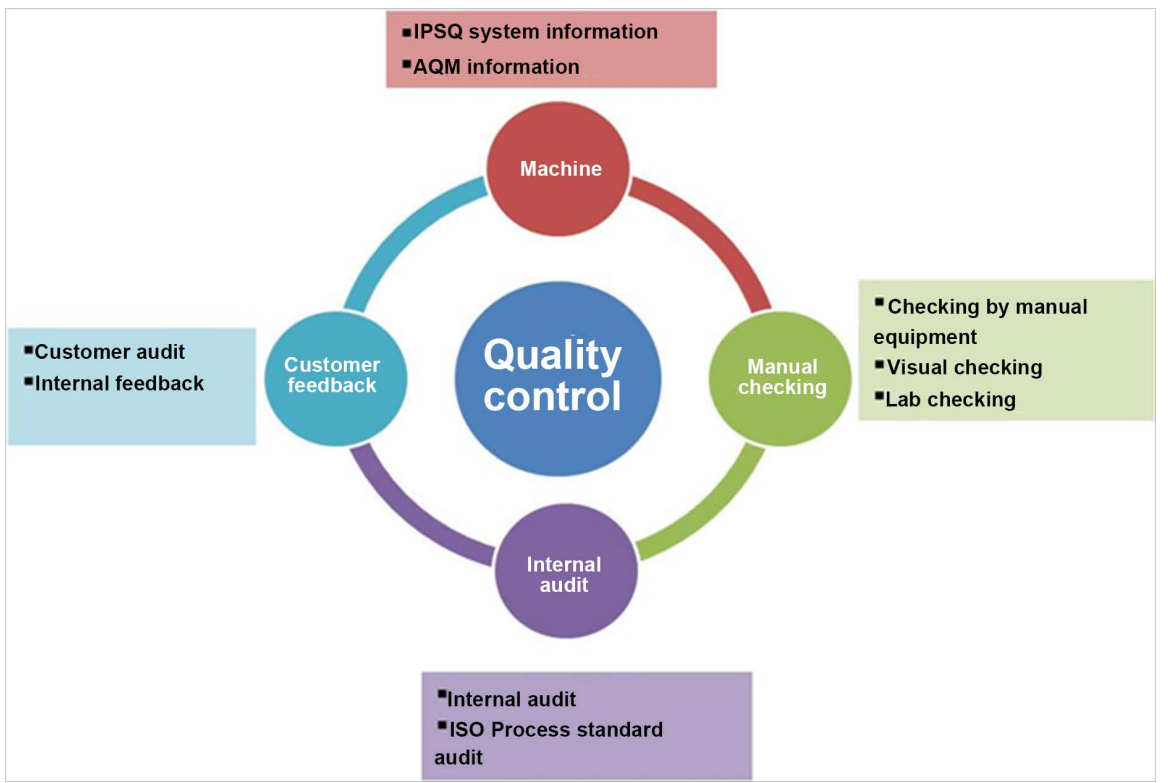

Figure 3. Quality control methods in the painting process.

internal target as hourly put, repair rate which helps us to figure out root cause in case of any tolerance of the production (Figure 4).

\section{Methodology}

\subsection{Identify the Potential Defects through Painting Process}

The paint shop is one of the most complex production areas of the vehiclemanufacturer. From today's perspective, the most important paint application procedures can be found here, involving a relatively long process chain. In the paint shop, the highest demands are made on the functional and visual quality of the painting, on the productivity of the painting installations, and on the environmental compatibility of the processes [14] [21]. These are responsible for the 


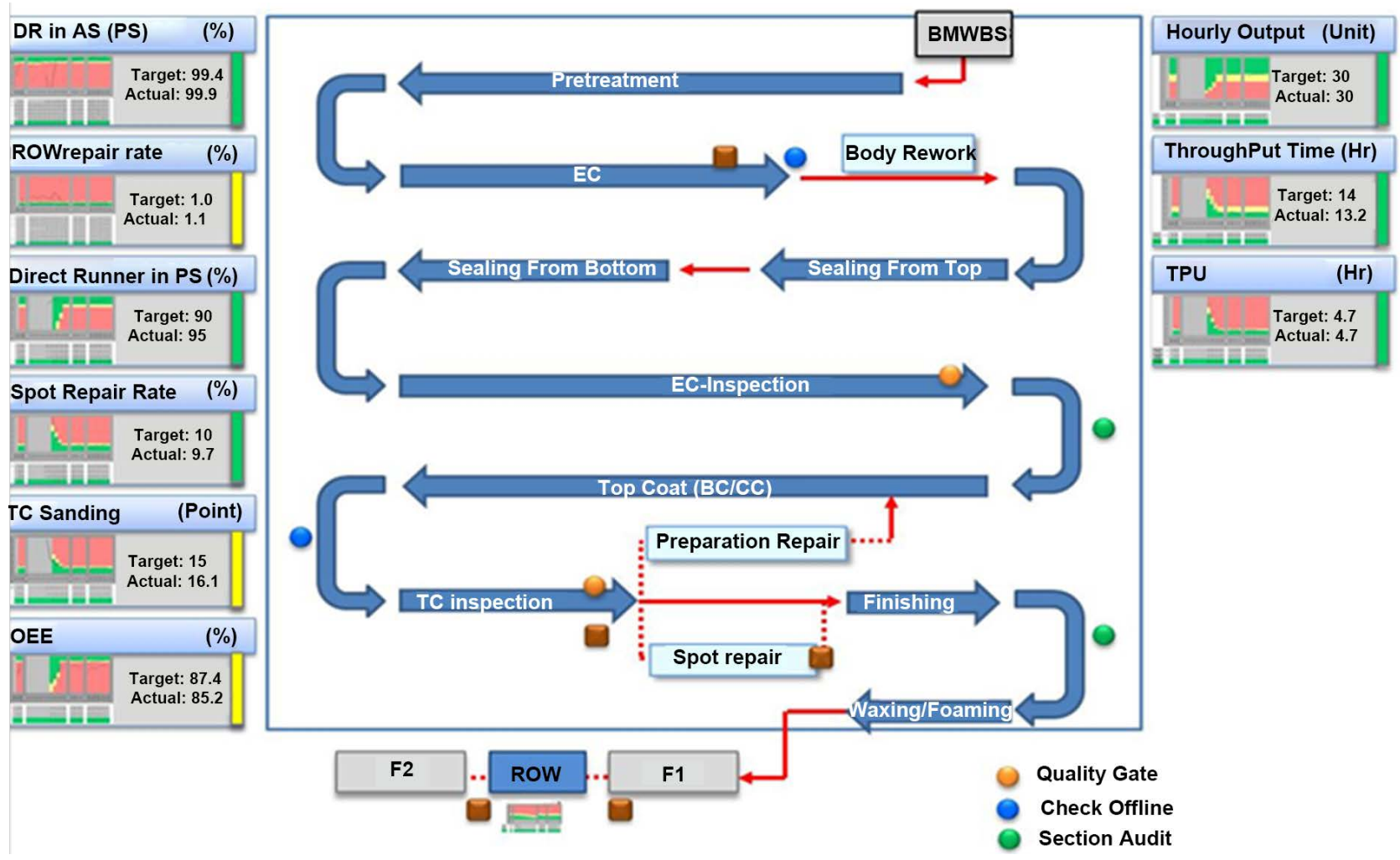

Figure 4. Quality control gate in the Painting process.

high degree of automation that can be found in automobile painting. In most paint shops, the individual coating processes are classified into coherent functional fields. They are arranged in such a way in the layout of the painting installation that a material flow results that is as simple and logical as possible, in relation to the connection of the paint shop to the neighboring production areas, the body shop, and the assembly line. A standard coating line for painting 60 units per hour is about $2 \mathrm{~km}$ long. The dwell time of a body is between 6 and 11 hours. About 30 - 50 people are employed per shift in a fully automated paint shop, mainly for maintenance, process control, and troubleshooting. The process chain includes value-adding and nonvalue-adding scopes of work. Nonvalue-adding jobs are typically manual jobs, for instance, repairs of body shop faults, sanding and polishing, cleaning, smoothing, and repainting. A future objective is to eliminate non-value-adding jobs completely, or at least reduce them to the minimum extent possible. Value-adding processes have reached a high degree of automation today and it is expected that full automation will be achieved in the future. The increasing pressure for reduction in costs is reflected in the effort to reduce the cost per unit (CPU). This has led to innovations in the customer-supplier relationship and in the painting process. The standard painting process, which has been used for years by all Original Equipment Manufacturers (OEMs), consists of the steps primer, base coat 1, basecoat 2, and clear coat. Consolidated processes are now being introduced which involve shorter process times, where either the primer application is dispensed with, or where all 
coats are applied wet-on-wet, without intermediary drying (see Figure 5). Surface coating technology is going through an exciting time. The purpose here is a clear-cost reduction, environmental compliance, and improved quality [9]. Car painting process methods are:

$>$ Pretreatment;

Electro coating;

Sealing and underbody protection;

$>$ Paint application;

Function layer and Basecoat application;

Clear coat application;

Cavity and wax application.

Pretreatment consists of the steps of precleaning, degreasing, purging, and phosphating. Precleaning removes the rough contaminations. Degreasing solubilizes grease, for example, deep-drawing greases, oil, wax, and other contaminations acquired from the earlier working processes [16]. Phosphating following after a purging process serves as a temporary corrosion protection and improves the adhesiveness of the paint film when it is applied. Electrocoat paints are water-soluble with only low proportions of organic solvents (approximately 3\%). Electrocoating covers all dip painting processes, where the paint precipitates on the workpiece owing to chemical conversion and associated coagulation of the binder [22]. The overlapping, spot-welded metal sheets must be sealed in such a way that no humidity can penetrate between the metal sheets and water in the vehicle interior, which may lead to corrosion there. On the weld seams, high viscous Polyvinylchloride (PVC) material is mostly sprayed as paths with the airless application or extruded by flat stream nozzles. The underbody protection also serves as protection from corrosion, mostly for areas exposed to a high strain because of stone chips. It is applied partially two-dimensionally, for instance, in wheel arches, and in the rocker panel area [23]. The primer surfacer which is called $\mathrm{BC} 1$ applied on top of the electro coat protects the cataphoretically

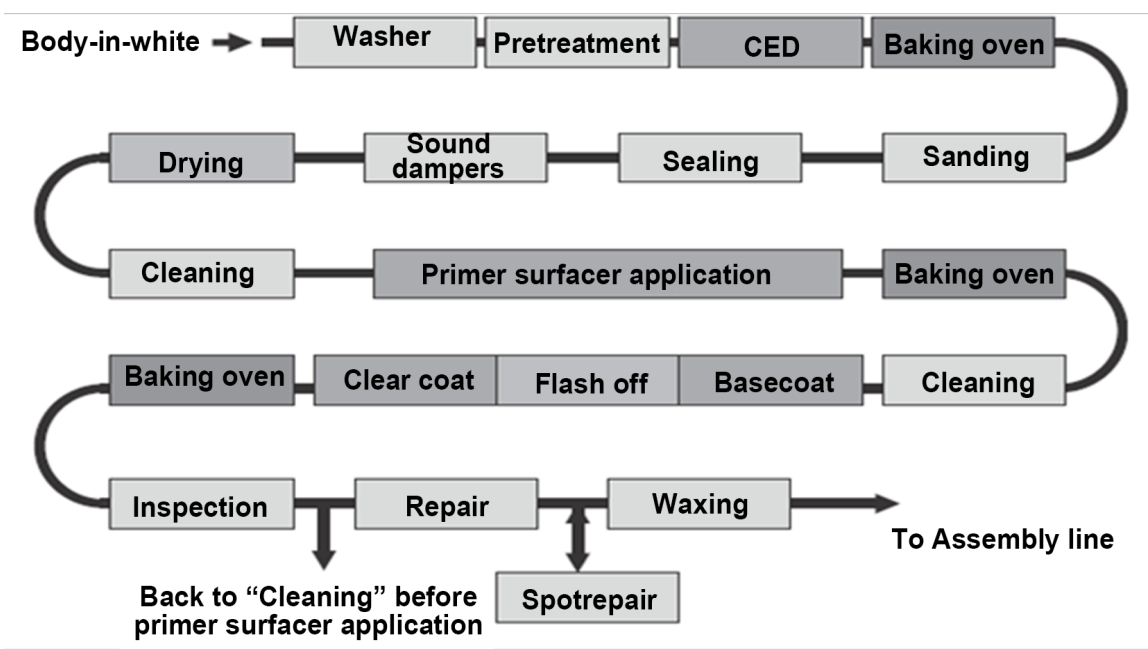

Figure 5. Process steps in modern automotive paint shops. 
cataphoretically electrocoating film from ultraviolet (UV) radiation, serves as a surface smoothing primer for the following topcoat film, and reduces the risk of damage to the layers below, in case of stone chips. Bumps and faults stemming from the body shop like grinding remains can be repaired by sanding the primer coat. The primer is applied to the high-speed-rotating application with the electrostatic charging of the paint material. For reasons of volatile organic compounds (VOC) emission, hydro primer materials are mostly used in Europe and powder, to a certain degree, in North America. A further reduction of emissions has been achieved with the development of two different processes.

The function layer combines the characteristics of the primer and the base coat material. It is matched in color to the following basecoat material, which is then only applied in one coat on the wet or flashed off function layer. This is also valid for the metallic effect material. In this process, the entire process chain consists only of four paint applications cathodic electrocoating, function layer, base coat, and clear coat, compared to five layers for the conventional metallic painting cathodic electrocoating, primer surface, basecoat 1 , base coat 2, and clear coat. Apart from the emission reduction, this process has the advantages of the reduced installation investment and overheads owing to the omission of one painting line. A disadvantage is the fact that faults from the body shop and the cataphoretic electrocoat process that are not eliminated after the cataphoretic electrocoat can only be processed after the top coat painting. The top coat is applied after athorough cleaning of the entire car body. The prevalent process for the top coat application is the application of a waterborne base coat, followed by a clear coat. The topcoat application is followed by a quality control check. The paint film is examined for faults like dirt inclusions, wetting disturbances, runners, and other such defects. Additionally, the film thickness and the visual parameters like color shade, gloss, and leveling are measured regularly. The corrosion protection measures are finalized with the sealing of the cavities with wax materials. For this, two procedures are usually followed-spraying and flooding [3]. For spraying, special nozzles are inserted in the cavities, and an exactly measured quantity of material is sprayed inside each cavity. For flooding, the cavities are filled with flooding wax, under pressure.

\subsection{Process related Factors of Paint Performance}

The reliable, objective, and reproducible measurement of the quality-relevant data like film thickness, color shade and leveling are absolute requirements for a quality-oriented process-control system. The film thickness distribution on a surface is subject to a more or less distinct fluctuation that is caused by many factors. The main factors are the application technology, the setting of the atomizer parameters, the number of overlaps of the individual spraying paths and spray-booth conditions [3]. On the paint surface, there are various factors that can influence the paint quality. According to lab analysis, paint surface affected by the process parameter, environment, dosing factors, equipment, and manual 
operational. Paint function defects evolution standard for process parameters factors (Table 1).

\subsection{Quality Aspects of the Painting Process}

The management level of a paint shop requires constantly updated data and facts about the state of the painting installation, to be able to make decisions based on the facts available. These may be short-term decisions such as the introduction of extra shifts, or changes in operations owing to a high repair rate, or long-term measures like a change of a subcontractor owing to quality problems [24]. The system accesses the data from the process data recording and consolidates them to the required management data. The data feedback from the different module which is depending on the machine, manual checking, internal audit and customer feedback [25]. Quality manual inspection is carried out by a different measuring device such as color measurement device, film build device, appearance measuring device, gloss measuring device. Aside Quality online measuring system AQAMis controlled by the trained qualified specialist which data monitor by the quality database and processed immediately and shows on the workpiece, according to their place of measurement. IPSQ system uses to input the defects online during the manual operation by the qualified operator.

The results of the automotive coating surface measurement data which control the surface defects as film build, paint structure, color measurement. All this three-important surface factors are measuring by the robots' technology and get an initial result of the car surface and can see the color deviation from the standard. The measuring requirement is according to the production batch of the color and if there some non-conforming product found initially test a certain amount of car to figure out the defective cars and analysis the measuring data by the responsible engineers [18]. In order to make sure the data of the automotive measuring equipment is in control also measuring car with the manual device to compare with AQM data in order to make sure the capability of the process performance is in range. The Automatic painting installations with units like

Table 1. Defects type with surface description.

\begin{tabular}{|c|c|c|c|c|}
\hline Type of defects & \multicolumn{4}{|c|}{ Defects description } \\
\hline \multirow{2}{*}{ Color tolerance } & \multicolumn{4}{|c|}{$\mathrm{DE} \leq 1.4($ good $), \mathrm{DE}<1.7$ (Accepted $)$} \\
\hline & \multicolumn{4}{|c|}{$\mathrm{DE}>1.7$ out of tolerance } \\
\hline \multirow{6}{*}{ Paint Appearance } & \multicolumn{2}{|c|}{ Horizontal } & \multicolumn{2}{|c|}{ Vertical } \\
\hline & N1 & N3 & N1 & N3 \\
\hline & $<6.0$ & $<6.0$ & $<3.2$ & 3.7 \\
\hline & $6.0<\mathrm{X}<7.0$ & $6.0<\mathrm{X}<7.0$ & 3.2. $<\mathrm{X}<4.2$ & 3.7. $<\mathrm{X}<4.7$ \\
\hline & $7<\mathrm{x}<8$ & $7<x<8$ & $4.2<x<5.2$ & $4.7<\mathrm{x}<5.7$ \\
\hline & $\geq 8$ & $\geq 8$ & $\geq 5.2$ & $\geq 5.7$ \\
\hline Film build & Horizontal & $\begin{array}{c}\leq 90(\text { bad }) \\
>100 \leq 125 \\
\quad(\text { good }) \\
>125(\text { bad })\end{array}$ & Vertical & $\begin{array}{c}\leq 90(\text { bad }) \\
>100-<120(\text { good }) \\
>120(\text { bad })\end{array}$ \\
\hline Gloss & \multicolumn{4}{|c|}{ DOI > 90 (good) } \\
\hline
\end{tabular}


process equipment, environmental equipment, conveyors, robots, and application equipment are complex systems whose controllability is subject to the performance of the total process and the coating result [12]. Therefore, the functionality and the operability of the control-technology equipment are essential, and high demands are made on the appropriate control technology.

\subsection{Process Monitoring and Regulation}

The complexity of automatic painting installations and a large number of different process parameters makes it complicated for the plant operator to maintain a high quality of production, and for the service personnel to eliminate defects without delay [26]. Systems that support the operator in the diagnosis, optimization, and monitoring of the processes are already in use. New systems are being developed to further improve on these, by taking into consideration quality-oriented control of the painting processes and process parameters. The linking up of such systems with all levels of the process and the installation, and with the control technology necessary for doing so, makes them very effective tools. As shown in Figure 6, the structure of the quality and process control methods mainly contains three steps:

Step 1: Function step: It makes real-time data acquisition and analysis in the manufacturing process. It can output the statistical analysis results in the form of a quality report. Input the data of manufacturing process into the SPC system [4]. Data mainly collect on the basis of the Manual inspection, Audit feedback (internal and external audit), automotive measuring feedback (AQM, IPSQ system).

Step 2: Data evaluation: It implements the FMEA process, which conducted by the experts coming from the different area. The knowledge and experience of experts will be extracted by the brainstorming activity. Then, The FMEA results will be transformed into FMEA knowledge according to with a specific method and put into FMEA repository [11].

Step 3: Risk Priority: It's designed to store the data collected by SPC system and find out the potential root cause after priorities the specific repository and take immediate control plan and corrective action in order to the improvement of the quality [19].

\section{Results and Discussion}

\subsection{Analysis and Interpretation}

In order to get the defects frequency rate, use the SPC control chart. Its help to figure out the highest frequency of the defects that influence the process during the production. Figure 7 shows the defect frequency range within five calendar weeks. Calendar week 1 shows that among the defects inclusion is one of the most higher falling rates per unit and its one of the reason for the scraping of the car and influence the TAKT time during the production and its direct influence the production target [27]. In the TOP coat line each car defects setting target is 


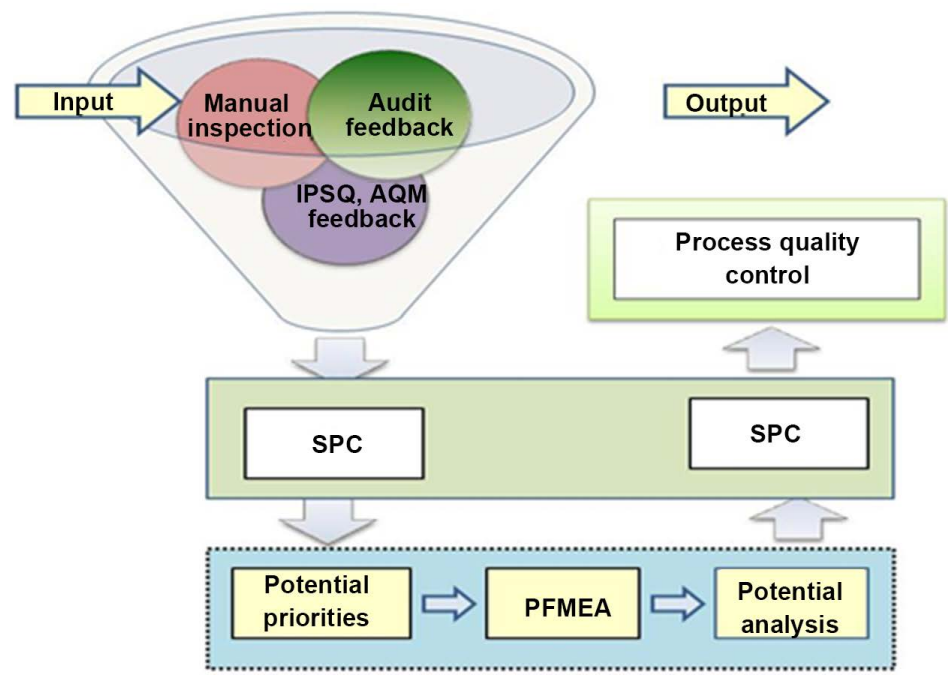

Figure 6. A specific control based on FMEA repository and SPC system.

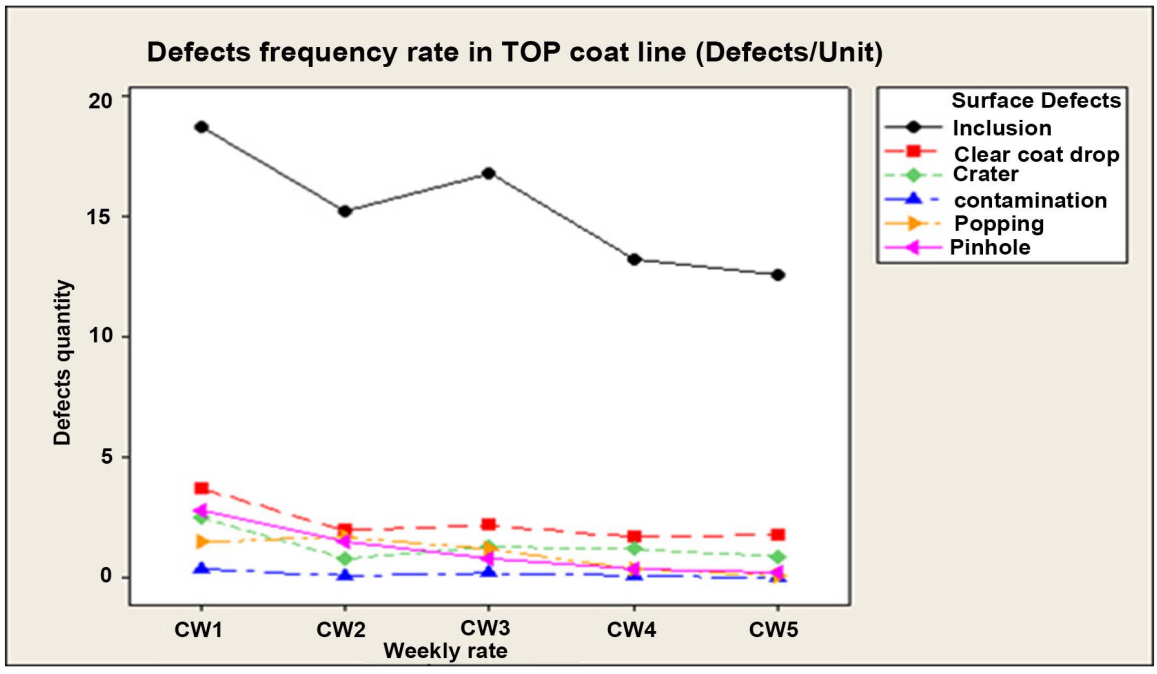

Figure 7. Diagram of the weekly percentages of the non-conformities.

10 defects but from the data, we can see that inclusion defects mostly influence the target. To analysis, the defective car from the total production, Take the sample as a 5 subgroup and each group check the 10 cars as a sample.

Pareto charts are a graphical demonstration of the occurrences, with the most frequently occurring event to the left and less frequent occurrence to the right. The Pareto charts in Figure 8 shows the occurrences of defects in a painting process organization. $78 \%$ of the defects in the surface are inclusion, followed clear coat drop at $16 \%$. The from the chart can see that these two types of defects are the most prevalent. In the final inspection line, a certain number of cars are rejected due to inclusion scratches, chips, bends, clear coat drop, popping or dents [24]. In order to evaluate the defects frequency use Pareto chart to see which defect is causing most of the problems. The operator checks each car surface and put the information into the IPSQ system. Figure 8 shows that inclusion is one of the top problems on the car surface. 


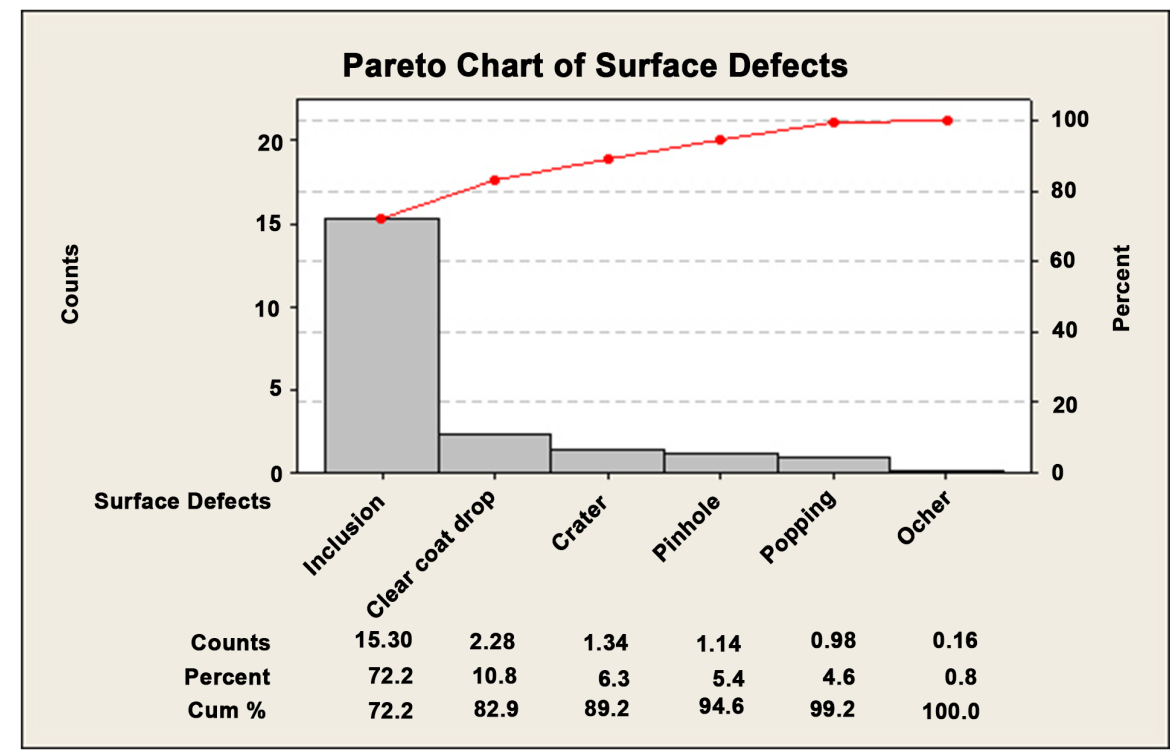

Figure 8. Pareto diagram for the surface defects trends in the TC.

Ishikawa analysis to figure out the potential causes of the inclusion defects:

These diagrams depict an array of potential causes of quality problems. The problem (the head of the fish) is displayed on the right, and the bones of the fish-representing the potential causes of the problem-are drawn to the left. Potential causes are often categorized as materials, equipment, people, environment, and management. Other categories may be included as appropriate. Useful in brainstorming the causes of problems from multiple perspectives, these diagrams should include all possible reasons for a problem. When completed, further analysis is done to identify the root cause. Figure 9 is an Ishikawa diagram to figure out the root cause of the particle issues in the top coat line. From this issue need to prioritize the possible causes that may influence the inclusion defects on the car surface [12]. To eliminate this defect, first need established a team which integrated with the relevant departments as a core team.

\subsection{Potential Root Cause and Risk Analysis by FMEA}

FMEA method is applied in painting technological process, so the severity (SEV) of risk occurrence, the probability (OCC) of risk occurrence and the probability of risk detection (DET) are determined. All assessments are expressed by numerical values. The numerical value can be calculated the value of Risk Priority Number (RPN) with Equitation (2):

$$
R P N=O \times S \times D
$$

The ranking of RPN is presented in Table 2.

FMEA worksheet creation, there have been used data from Department for control and quality assurance, Maintenance department for equipment interventions, Production Department and IT department.

FMEA is made in each step of the production process of the paint shop, and there are determined some potential causes of failure function in a separate 


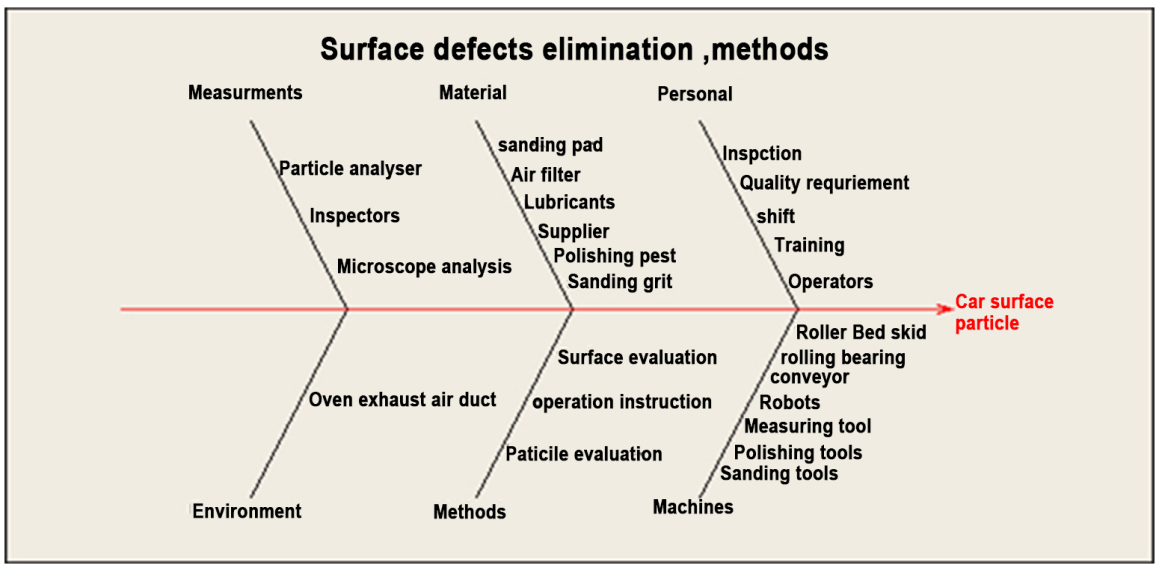

Figure 9. Ishikawa diagram prepared for investigation of the cause of the particles on surfaces.

Table 2. A general indication of the risk.

\begin{tabular}{cc}
\hline Value of RPN & Evaluation of the risk \\
\hline 100 & Significant \\
$10<$ RPN $<100$ & Less significant \\
$<10$ & Negligible
\end{tabular}

phase. FMEA analysis shows that RPN has a higher value of 245 in the process of manual inspection of the car surface, RPN rate is 126 in the process steps roller bed and skid inspection and Lab test results process steps RPN is 128. As the risk value priority is higher of 100 , the risk in the 1st, 3rd and 5th step (the process of inspection, roller, and skid, lab test) from the production process is considered as significant, while in the rest of the steps the value of the RPN ranges between 8 - 100 [1]. Therefore, it is considered as less significant or insignificant. If surface inclusion is determined during the control measurement of the inspection process and small opportunities for the detection i.e. $\mathrm{DET}=5$. While in the daily roller bed and skid inspection process and lab test process the fault is obvious and the probability that the product with defects will be distributed is low (DET $=2-3$ ). The value of RPN shows in plot chart (Figure 10), shows that the 1st, 3rd and 5th process of the diagram are considerably higher in relation to the other phases and after take action the RPN rate is in control rate and mean value is 5.8 that is negligible according to the RPN rating scale. So, the process is in the control limit.

\subsection{Reflow SPC Measurement Data}

To verify that the process has been improved and need to analyze that how efficient the FMEA during the production process, it's necessary to reevaluate the data falls on the TOP coat line. The Figure 11 shows that after RPN action improvement inclusion trends are going down 18 to the 5 defects in the car surface 


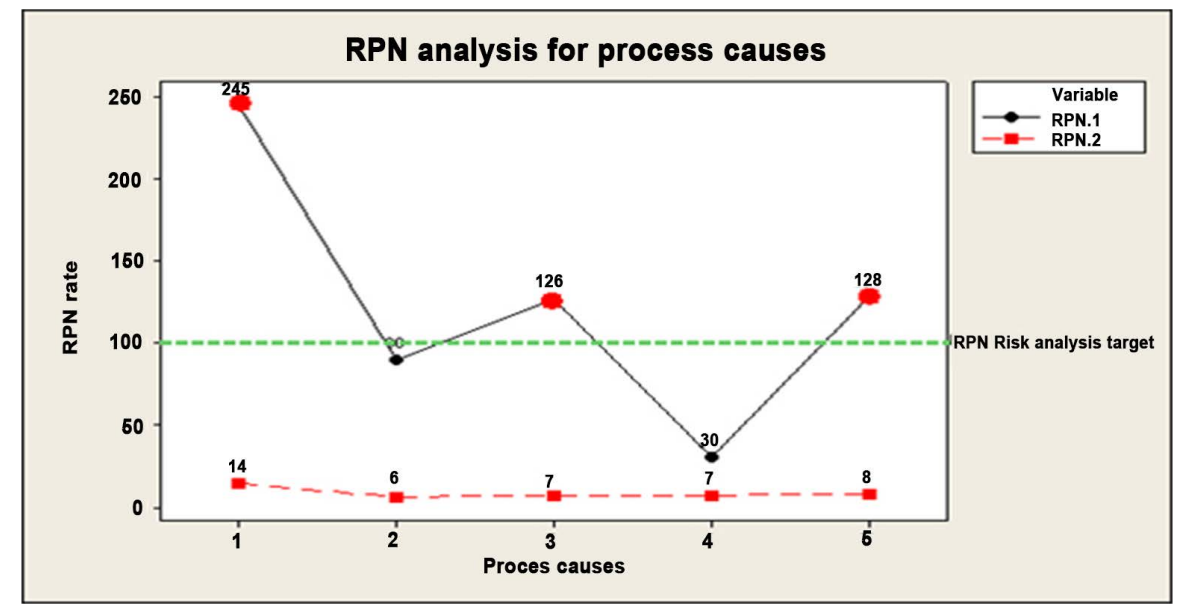

Figure 10. Lin plot chart for evaluation the RPN rate before and after the corrective action.

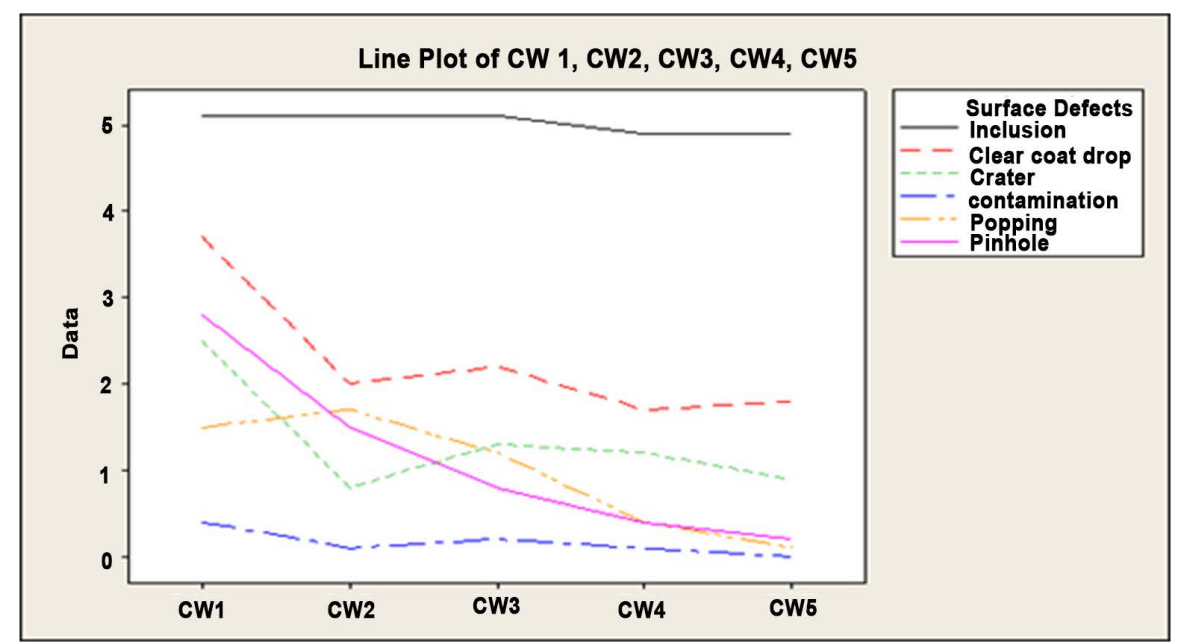

Figure 11. Diagram of the weekly percentages of the non-conformities after corrective action.

which can easily understand that new improvement actions could be proposed toward the minimization of the RPN. From the Figure $11 \mathrm{C}$-chart shows that average inclusion trends rate is 3.94 which are under the control. The data is a collectionof the corrective action taken which improvement is obvious.

\subsection{Evaluation}

FMEA is a very effective risk analysis method for a company but it is not obligatory to use but if any organization uses it must get several benefits as it is mentioned in this report. In an automotive company, they use only Design and Process FMEA and some qualitative part of criticality analysis. To complete an FMEA analysis, it is necessary to make a cross-functional group from different departments of the company. The team will be composed of experienced and devoted person will search for failure mode, cause, effect, severity, occurrence, and detection. Brainstorming is very necessary for this FMEA worksheet. It is 
also required to find the proper way to lessen the failure mode. Severity ranking remains almost same if the failure mode is not eliminated. In FMEA worksheet, if severity ranks 10 or 9 , it shows red mark (red marks suggest for quick preventive work). There will be an acceptable RPN limit for any company. It may differ for different companies. Painting process has a grand limit of 100 RPN. The FMEA team needs to see after the action was taken for the design or process whether the RPN value is less than 100 or not. One will get a graph of RPN of before (red marked) and after the action (blue marked). Here, it is possible to compare the performance development by FMEA process. It is seen that before the action was taken the RPN value was 150 - 260 but when the corrective action was taken the RPN values plunged exponentially from 150 - 260 to 30 - 100 [23]. If it is not less than 200, the FMEA team is instructed to take necessary corrective action and will have to compare the RPN value of before the action was taken and after the action was taken. In criticality analysis, the occurrence data are plotted in $\mathrm{X}$-axis and severity data are plotted in $\mathrm{Y}$-axis. As a result, there are four zones for considered according to the position of failure modes named: confirmed critical characteristics confirmed significant characteristics, RPNTop 20\% by Pareto and annoyance region [28]. From these zones, the FMEA team can decide that which failure modes should be prioritized more. The zones should be considered confirmed critical characteristics zone, confirmed significant characteristics, annoyance region and RPN-Top 20\% by Pareto respectively. RPN-Top $20 \%$ by Pareto means which $20 \%$ failure mode should be prioritized of $100 \%$. Top 20\% failure modes should be considered as the most part of the zone is very acceptable. The study shows some differences between FMEA and FMECA. The main difference between the company findings and the theoretical finding of this report is: Parker Hannifin is using a grand limit for RPN value and it is 200 [24]. If severity ranks 10 or 9 , it marks red for alarming the design or process. In criticality analysis, the company is only performing the the qualitative part.

\section{Conclusion}

The application of an FMEA reveals the hidden process weaknesses, leading to the quantification of failure related indicators/failure risks and the creation of a prioritization matrix for further improvement actions. Risk reassessment and further preventive action planning could lead to effective risk minimization. The use of an FMEA can also be applied successfully in various other business sectors (e.g., supplies, sales, financial), leading to continual improvement and increasing the bottom-line results. After execution of the process FMEA for inclusion defects problem, it is clear that FMEA is a most useful tool to identify potential failures reduces those effects by implementing control plans. Hence it can be heavily improving the quality of the product and enhance product performance. FMEA execution is an only present potential failure and asks to implement preventive measure to stop the occurrence of failure and enhance product and process performance so it identification and implementation of prevention tech- 
nique for potential failure is very important. The application of appropriate methods and techniques for monitoring and quality control in the painting process will allow managers scientific approach in the quality assurance and production of high-quality products at minimum costs. Based on the results of FMEA and Pareto analysis, the source of poor quality is identified and its reduction of $80 \%$ of the top 1 defects. The corrective action is taken and the required accuracy in top coat line is achieved with the combination of the FMEA and SPC method figures out the excellent tools for organizations self-assessment and tends to improve the performance of top coat defects.

\section{References}

[1] Akafuah, N., Poozesh, S., Salaimeh, A., et al. (2016) Evolution of the Automotive Body Coating Process-A Review. Coatings, 6, 24. https://doi.org/10.3390/coatings6020024

[2] Sarker, M.N.I., Hossin, M.A., Anusara, J., et al. (2018) Practices of Low Carbon Economy in China: Challenges and Opportunities for Economic Development. Low-Carbon Economics, 9, 18-32. https://doi.org/10.4236/lce.2018.91002

[3] Poozesh, S., Akafuah, N. and Saito, K. (2018) Effects of Automotive Paint Spray Technology on the Paint Transfer Efficiency-A Review. Journal of Automobile Engineering, 232, 1-20. https://doi.org/10.1177/0954407017695159

[4] József, B. and Blaga, P. (2017) Optimizing Production Costs with the Correct Setting of the Paint Thickness Parameters. Procedia Engineering, 181, 1021-1027. https://doi.org/10.1016/j.proeng.2017.02.502

[5] Pophaley, M. and Vyas, R.K. (2010) Plant Maintenance Management Practices in Automobile Industries: A Retrospective and Literature Review. Journal of Industrial Engineering and Management, 3, 512-541.

[6] Sarker, M.N.I. and Jie, Z. (2017) Social Security for Vulnerable Groups in Bangladesh on Government Perspective: Contribution of Research Leader. Journal of Public Administration and Policy Research, 1, 1-9.

[7] Kamani, P., Noursadeghi, E., Afshar, A. and Towhidkhah, F. (2011) Automatic Paint Defect Detection and Classification of Car Body. 2011 th Iran Conf Mach Vis Image Process MVIP.

[8] Sarker, M.N.I., Hossin, A., Yin, X. and Sarkar, M.K. (2018) One Belt One Road Initiative of China: Implication for Future of Global Development. Modern Economics, 9, 623-638. https://doi.org/10.4236/me.2018.94040

[9] Sarker, M.N.I., Hossin, M.A., Hua, Y.X., et al. (2018) Low Carbon City Development in China in the Context of New Type of Urbanization. Low Carbon Economy, 9, 45-61. https://doi.org/10.4236/lce.2018.91004

[10] Haider, M.K., Islam, M.S., Islam, S.S. and Sarker, M.N.I. (2015) Determination of Crop Coefficient for Transplanted Aman Rice. International Journal of Natural and Social Sciences, 2, 34-40.

[11] Yeh, T.M. and Chen, L.Y. (2014) Fuzzy-Based Risk Priority Number in FMEA for Semiconductor wafer Processes. International Journal of Production Research, 52, 539-549. https://doi.org/10.1080/00207543.2013.837984

[12] Arinez, J., Biller, S., Meerkov, S.M. and Zhang, L. (2010) Quality/Quantity Improvement in an Automotive Paint Shop: A Case Study. IEEE Transactions on Automation Science and Engineering, 7, 755-761. 
https://doi.org/10.1109/TASE.2009.2033568

[13] Judi, H.M., Jenal, R. and Genasan, D. (2011) Quality Control Implementation in Manufacturing Companies: Motivating Factors and Challenges. In: Ivanov, O., Ed., Applications and Experiences of Quality Control, InTech Europe University Campus, Rijeka, 495-508.

[14] Li, J., Blumenfeld, D.E. and Marin, S.P. (2007) Manufacturing System Design to Improve Quality Buy Rate: An Automotive Paint Shop Application Study. IEEE Transactions on Automation Science and Engineering, 4, 75-79. https://doi.org/10.1109/TASE.2006.872958

[15] Arabian-Hoseynabadi, H., Oraee, H. and Tavner, P.J. (2010) Failure Modes and Effects Analysis (FMEA) for Wind Turbines. International Journal of Electrical Power \& Energy Systems, 32, 817-824. https://doi.org/10.1016/j.ijepes.2010.01.019

[16] Sarker, N.I., Hossin, A., Hua, Y., et al. (2018) Oil, Gas and Energy Business under One Belt One Road Strategic Context. Open Journal of Social Sciences, 6, 119-134. https://doi.org/10.4236/jss.2018.64011

[17] Bharatbhai, M.G. (2015) Failure Mode and Effect Analysis of Repower 5M Wind Turbine. International Journal of Advanced Research in Science, Engineering and Technology, 2, 2394-2444.

[18] Darrall, K.G., Pindar, A., Quevauviller, P. and Interlaboratory, P. (1997) Collaborative Study for the Quality Control of Trace Element Determinations in Paint Coatings. Fresenius Journal of Analytical Chemistry, 357, 833-836.

https://doi.org/10.1007/s002160050258

[19] Simões, D., Daniluk Mosquera, G.A., Batistela, G.C., et al. (2016) Quantitative Analysis of Uncertainty in Financial Risk Assessment of Road Transportation of Wood in Uruguay. Forests, 7, 1-11. https://doi.org/10.3390/f7070130

[20] Mcintee, E. (2008) Forensic Analysis of Automobile Paints by Atomic and Molecular Spectroscopic Methods and Statistical Data Analyses.

[21] Sarker, M.N.I. (2017) Smart City for Smart Citizen: Dimensions, Challenges and Opportunities for Developing Country. 5th Smart Cities Conference, Bucharest, 38.

[22] Bookbinder, J.H. (2015) Dynamic Control Rules for Conveyor Intersections in a Truck-Assembly Paint Shop. The International Journal of Advanced Manufacturing Technology, 76, 1515-1527. https://doi.org/10.1007/s00170-014-6235-3

[23] Salihoglu, G. and Salihoglu, N.K. (2016) A Review on Paint Sludge from Automotive Industries: Generation, Characteristics and Management. Journal of Environmental Management, 169, 223-235. https://doi.org/10.1016/j.jenvman.2015.12.039

[24] Ju, F., Li, J., Xiao, G. and Arinez, J. (2013) Quality Flow Model in Automotive Paint Shops. International Journal of Production Research, 51, 6470-6483. https://doi.org/10.1080/00207543.2013.824629

[25] Sarker, M.N.I., Bingxin, Y., Sultana, A. and Prodhan, A.S. (2017) Problems and Challenges of Public Administration in Bangladesh: Pathway to Sustainable Development. International Journal of Public Policy and Administration Research, 3, 16-25.

[26] Jones, K., Cocker, J. and Piney, M. (2013) Isocyanate Exposure Control in Motor Vehicle Paint Spraying: Evidence from Biological Monitoring. Annals of Occupational Hygiene, 57, 200-209.

[27] Sellappan, N. and Palanikumar, K. (2013) Modified Prioritization Methodology for Risk Priority Number in Failure Mode and Effects Analysis. International Journal of Applied Science and Technology, 3, 27-36. 
[28] Quevauviller, P. (2001) Certified Reference Materials for the Quality Control of Inorganic Analyses of Manufactured Products (Glass, Polymers, Paint Coatings). TrAC Trends in Analytical Chemistry, 20, 446-456.

https://doi.org/10.1016/S0165-9936(01)00090-5 